\title{
Traduire
}

Une autre perspective sur r tr traduction

Revue française de la traduction

227 | 2012

Éco, socio, philo... \& co

\section{Bernard Simeone. Traducteur de Luzi, Caproni et Sereni en France. Antonino Velez}

\section{Sophie Guermès}

\section{(2) OpenEdition}

10 Journals

Édition électronique

URL : http://journals.openedition.org/traduire/499

DOI : $10.4000 /$ traduire.499

ISSN : 2272-9992

Éditeur

Société française des traducteurs

\section{Édition imprimée}

Date de publication : 15 décembre 2012

Pagination : 117-120

ISSN : 0395-773X

\section{Référence électronique}

Sophie Guermès, "Bernard Simeone. Traducteur de Luzi, Caproni et Sereni en France. Antonino Velez »,

Traduire [En ligne], 227 | 2012, mis en ligne le 24 avril 2014, consulté le 22 septembre 2020. URL

http://journals.openedition.org/traduire/499 ; DOI : https://doi.org/10.4000/traduire.499 


\title{
Bernard Simeone. Traducteur de Luzi, Caproni et Sereni en France. Antonino Velez
}

\author{
Sophie Guermès
}

\section{RÉFÉRENCE}

Bernard Simeone. Traducteur de Luzi, Caproni et Sereni en France (Palermo, Herbita editrice, $2012,16,50 €$ - en vente sur Internet)

1 Dans ce volume, Antonino Velez s'attache à retracer le travail de traducteur de Bernard Simeone, en choisissant de se focaliser sur la traduction de trois écrivains italiens majeurs, qui partageaient avec Bernard Simeone la particularité d'être à la fois poètes et eux-mêmes traducteurs : Mario Luzi, Giorgio Caproni, et Vittorio Sereni.

2 Ces trois poètes font partie de ce qu'on a coutume d'appeler la " troisième génération ", génération qui a succédé à celle d'Ungaretti, Saba, et Montale. Caproni et Sereni ont aussi en commun d'avoir traduit René Char (Caproni, une sélection de poèmes et de proses, en 1962, Sereni, Feuillets d'Hypnos en 1968, Retour amont et autres poèmes en 1974). C'est justement un extrait de Feuillets d'Hypnos, "Épouse et n'épouse pas ta maison », que Bernard Simeone avait placé en épigraphe d'Acqua fondata, publié en $1997^{1}$.

Bernard Simeone, qui a souvent traduit des poètes vivants (certains appartenant à sa propre génération), a connu Luzi, Caproni et Sereni, comme beaucoup d'autres, qu'il évoque notamment dans Acqua fondata (Attilio Bertolucci par exemple, autre représentant de cette "troisième génération", et qui avait choisi, comme Giorgio Caproni, de vivre à Rome dans le quartier Monteverde).

4 Si A. Velez a choisi Luzi, Caproni et Sereni, c'est qu'il juge - et il le montre en développant plusieurs exemples - qu'une "lecture attentive des vers» de B. Simeone "met en évidence l'influence» de ces trois poètes qu'il a traduits parmi beaucoup d'autres. «Cet apport ne peut être réduit à de purs aspects rythmiques ou stylistiques, 
mais contient une vision globale et commune de la vie et de l'art. » (p. 42) B. Simeone partageait notamment avec Mario Luzi la conception de la poésie comme «éternel exercice d'approfondissement ".

Dans la première partie de son livre, A. Velez retrace l'itinéraire de traducteur de $B$. Simeone, marqué par l'abondance de la production autant que par l'exigence, dans le choix des auteurs et des textes comme dans le choix des mots. La traduction était pour B. Simeone un engagement existentiel : « Pour que je traduise », écrivait-il en 1987, « il faut que le poème en italien m'habite, m'obsède. » (cité p. 23). Et l'on est frappé par la justesse de l'exercice (A. Velez en donne de nombreux exemples, concernant les trois poètes qu'il a choisi de privilégier) en même temps que par le "travail immense accompli dans un laps de temps relativement court » (p. 20). Travail dont la très longue, sans doute exhaustive bibliographie de fin de volume, permet en effet de mesurer l'ampleur.

6 En ouverture, A. Velez rappelle un souvenir qui lui est particulièrement cher, qu'il évoque au présent - et ce choix temporel suffit à exprimer la résonance intacte de ce moment, par delà le passage des années, et le deuil - : c'est celui d'un jour de novembre 1984, où B. Simeone et A. Velez sont réunis dans le bureau de Mario Luzi, à Florence. B. Simeone est alors un jeune traducteur - il commencera un an plus tard une collaboration fructueuse avec Philippe Renard, qui les mènera à fonder en 1987 la collection « Terra d'altri » aux éditions Verdier -, mais il est aussi l'auteur d'un premier roman, Figures de silence, publié à Lyon l'année précédente, et d'un récit, Courir à nouveau, qui vient alors de paraître; quant à A. Velez, il est étudiant à l'école d'interprètes de Trieste, mais déjà admiratif de l'écriture de B. Simeone (qu'il a rencontré à Villeurbanne en février 1984) puisqu'il a décidé de préparer une thèse sur Figures de silence; il est également passionné de traduction. Il traduira d'ailleurs Cavatine quelques années plus tard. Un peu plus loin (p. 39), A. Velez rapporte qu'après avoir lu Figures de silence, Philippe Renard signala à $\mathrm{B}$. Simeone les "crete» (crete senesi) décrites dans ses poèmes, et que Simeone avait lui-même évoquées dans son premier roman $^{2}$.

7 À cette époque, comme il le rappelle, la poésie italienne contemporaine commence à peine à passer les frontières : on la traduit depuis peu, et c'est Antoine Fongaro qui a été le premier à diffuser en France l'œuvre de Luzi, suivi en 1976 par Philippe Jaccottet. C'est à partir des années 80 que les traductions se multiplient, et, pour continuer à prendre l'exemple de Luzi, celui-ci devient en France, à partir de 1985, le plus lu des poètes italiens de sa génération, grâce à la traduction publiée chez Flammarion par Bernard Simeone et Philippe Renard, de trois recueils (Dal fondo delle campagne, Nel magma, Su fondamenti invisibili) regroupés en français sous le titre L'Incessante Origine.

8 Donc, avant d'étudier le travail de traduction accompli sur les poèmes de Luzi, Caproni et Sereni, A. Velez retrace le contexte dans lequel est arrivé B. Simeone en tant que traducteur. Il évoque aussi longuement sa façon de travailler : en collaboration avec Philippe Renard, travail à la fois « bicéphale » et solitaire; attachement à la présence du texte original en regard; système de «compensations contiguës» (p. 81) rendues possibles par la proximité des deux langues, italien et français; et traduction inséparable d'une réflexion sur cet acte. Toute l'œuvre de B. Simeone, qu'on ne peut pas dissocier, étant toujours création, témoigne d'un souci de compréhension toujours plus profonde, qui trouvera sans doute son "point de vérité », pour employer une expression de l'écrivain, dans Cavatine. Traduire, pour B. Simeone, c'était « accueillir », 
s'oublier soi-même pour laisser place à l'autre. "Il faut ", écrivait Simeone en 1992, " mourir un peu à soi-même et à son écriture (et, au-delà d'elle, à sa propre langue) pour dire qu'on a tenté de traduire.» (cité p. 24). A. Velez souligne (p. 40-41) que B. Simeone a commencé à écrire des poèmes en même temps qu'il devenait traducteur, et cette mise en relation des deux actes me paraît un aspect fondamental, indispensable à la restitution du sens de l'activité créatrice de B. Simeone. Sortir de soi, échapper à l'égocentrisme, tel est d'ailleurs le bilan tiré à la fin d'Acqua fondata. Le poète a aussi analysé le mouvement réciproque et paradoxal de révélation et de détournement, en jeu dans l'acte de traduire, où le traducteur comme le texte semblent - pour reprendre le titre d'un des plus grands livres de Nathalie Sarraute - entre la vie et la mort, puis courent le risque d'une perte d'identité. A.Velez évoque chez Simeone l'importance de la « mort du mot précédant la renaissance du sens de la parole dans la langue traduite " (p. 28), et, corrélativement, l'attention portée à ce qui se passe dans "l'intervalle »; loin de verser dans le lieu commun illusoire d'un décalque du texte premier, B. Simeone entendait « livrer une expérience où l'exigence est toujours confrontée à l'échec ou à la perte »; ce propos, de 1999, entre en résonance avec un extrait d'Acqua fondata, à propos d'un séminaire de traduction organisé par Franco Buffoni: "Nos textes renaîtront en italien après avoir traversé on ne sait quelle étendue, enjambé on ne sait quel gouffre, échappé à on ne sait quelle mort dans l'espace entre deux langues. » (p. 68). "D'un même geste ", écrit-il encore, « le texte traduit me révèle et me détourne, tout comme je le révèle et le détourne, lui, d'un même geste, le soumettant au risque de la métamorphose, qui devient son futur et le mien.» (ib. 151) Lorsque ce danger est assumé, la pauvreté - pour employer les mots de René Char - devient privilège : « La traduction", remarque A. Velez, "sert à dépasser non seulement les limites de sa propre écriture, mais aussi les limites de sa propre pensée » (p. 25). Ce qui fait sa valeur, c'est la « reconnaissance de l'autre» (p. 33), essentielle pour B. Simeone. Celui-ci était agnostique (contrairement à Luzi) et il ne semblait pas souffrir comme Caproni de la «mort » de Dieu, sa génération ayant d'autres préoccupations mais il avait conservé du christianisme cette dimension fondamentale.

9 Enfin, la traduction permet aussi de creuser une question qui ne peut jamais avoir de réponse définitive - d'où le fait que tous les poètes évoqués ici aient aussi été des traducteurs. Préfaçant sa traduction de 1962, Caproni écrivait : « Pourquoi, malgré les risques, ai-je traduit et essayé de traduire le poète Char ? (...) Si je savais répondre, je saurais définir la poésie de René Char. » Ce qui fait écho, dans la structure même du raisonnement, à un texte publié dans Recherche de la base et du sommet, où Char écrivait : « Si je savais ce qu'est Rimbaud pour moi, je saurais ce qu'est la poésie devant moi, et je n'aurais plus à l'écrire. »

\section{NOTES}

1. Sur Luzi, voir Acqua fondata, p.111-114; sur Caproni, pp.128-130 (et p.132, B. Simeone commente la photo de Ph. Morier-Genoud où René Char et Caproni posent côte à côte, et qui est 
reproduite dans le livre d'A. Velez); sur Sereni, p. 48. Sur la traduction en général, voir la section intitulée «Que traduire en traduisant?", pp. 150-151.

2. A. Velez nous fait aussi découvrir un beau texte inédit, qu'il a traduit, de Mario Luzi, en hommage à B. Simeone. Luzi y attirait notamment l'attention sur l'exceptionnelle profondeur de celui-ci : « Il se distingua aussitôt, sur la scène plutôt prévisible de sa génération, par l'épaisseur spéciale, de nature éthique et émotive, qu'il accordait au langage.» (cité p. 52). Ce texte aurait dû préfacer la traduction en italien, toujours par A. Velez, du premier recueil d'études de B. Simeone sur la littérature italienne, Lecteur de frontières - le projet n'a finalement pas vu le jour. 